\title{
LEXICAL AND SYNTACTIC AMBIGUITY IN HUMOR
}

\author{
Intan Nur Charina \\ Sanata Dharma University \\ intancharina.ic@gmail.com \\ DOI: https://doi.org/10.24071/ijhs.2017.010113 \\ received 27 March 2016; revised 24 March 2017; accepted 25 June 2017
}

\begin{abstract}
Ambiguity occurs when a sentence has more than one meaning. Ambiguity can be caused by the ambiguous lexicon in which one word has more than one meaning and it can also be caused because of the way the sentence is structure (syntactic). The context also determines whether the sentence can be interpreted differently and become ambiguous. Ambiguity often causes confusion, and has become one of the phenomena in language studies, especially semantics. This study investigates ambiguity in creating humors. The data were taken from electronic sources in forms of newspaper headlines, jokes, riddles and anecdotes. The number of the data collection includes 25 cases of ambiguity. 12 sentences were lexically ambiguous, while the other 13 sentences were syntactically ambiguous. The results showed that lexical ambiguity and syntactic ambiguity were the language devices used to create puns in humor. The results also suggested that the ambiguity could be an effective source of humor when it particularly involves dual interpretations in which one interpretation gives a serious meaning and tone, whereas the other interpretation gives a humorous meaning which is not likely to occur in normal contexts.
\end{abstract}

Keywords: lexical ambiguity, syntactic ambiguity, humor

\section{Introduction}

Sentences containing jokes and humors often find their humorous power through ambiguity apparent in the English language (Seewoester, 2009). Ambiguous sentences have more than one meaning and sense. The different meanings can elicit different and humorous senses in certain contexts. In many humors and jokes, this kind of wordplay is often used. Therefore, ambiguity is an important means of creating puns in jokes, or riddles. Ambiguity is also deliberately employed in many contexts to elicit a sense of wit and to make a sentence more attractive, or to make people curious about it. In some newspaper headlines and advertisement slogans, ambiguous sentences are sometimes used.

Ambiguity as a language device commonly used to create puns in humors gives some insights into how word play can manipulate the interpretation of meaning resulting in humorous and witty senses. This suggests that due to the multi interpretations, certain different senses of a single sentence can confuse the 
meanings and give different comprehensions which may elicit humors in certain contexts. There has been previous studies conducted to analyze this process, such as Duffy, Kambe, \& Rayner 2001, Giora 2003, Gorfein 2001, MacDonald, Pearlmutter, \& Seidenberg 1994, Tabossi 1988 and translation issues Antonopoulou 2004, Laurian 1992, Lew 1996, Ptaszynski \& Mickiewicz 2004, Zabalbeascoa 1996. These prior studies found that ambiguity is a source which is often used to create humor.

There are two types of ambiguity commonly used as the source of humors, i.e. lexical and syntactic ambiguity. The former one refers to ambiguity conveyed through polysemous words or homonymous strings, while the latter refers to phenomena of ambiguous word order, referential ambiguity, and prepositional phrase arrangement (e.g. Hirst, 1987: 131-162; Gibson \& Pearlmutter, 1994), for example. This study is limited to the discussion of those two kinds of ambiguity since they are the most employed types of ambiguity found in humors. The purpose of this study is to indentify the types of ambiguity used in the ambiguous sentences which elicit humors, whether it is lexical or syntactic ambiguity. The study will also investigate the process on how the pun is created due to the multi interpretations of the sentence by analyzing the meanings and senses that it elicits. The study attempts to explore ambiguity of sentence meanings and the interpretation of meanings in puns and humors.

\section{Theory}

Types of ambiguity which often create humorous meanings include lexical, and syntactic/structural ambiguity. Lexical ambiguity in humors refers to the ambiguity that occurs in lexical level which involves a manipulation of legitimate and meaningful morpheme/ lexeme that elicits a serious and humorous interpretation (Seewoester, 2009).

\section{Lexical and Syntactic Ambiguity as a Mechanism of Eliciting Humors}

This ambiguity utilizes homonymy, and polysemy in creating the ambiguity, and can involve word class change. Homonymy refers to the unrelated senses of the same phonological word (Kreidler, 2002). Homonymy can include homographs, senses of the same written word, and homophones, senses of the same spoken word. Polysemy is similar to homonymy because both deal with multiple senses of the same phonological word, but polysemy is invoked if the senses are judged to be related (Kreidler, 2002). below:

The examples of lexical ambiguity and the dual meanings can be shown

(i) I saw a tall tree outside the house.

From this single sentence, we can interpret two meanings because the lexeme 'saw' have more than one single meaning. 'Saw' can be the past tense of the verb 'see' and it can also belong to a different verb 'saw' which means to cut something using a saw. Although saw also can belong to a noun, meaning a tool for cutting woods (Longman Dictionary), the possible interpretations are limited to the verbs due to the syntactic (grammatical) requirement of the sentence. Thus the sentence can mean either 'I saw (past tense of see) a tree' or 'I saw (cut using a saw) a tree'. This ambiguity is classified into lexical ambiguity, because the dual interpretations are cause by the multiple meanings of the lexeme 'saw'. The 
contexts of the word occurrence also make it possible for the meanings to be both interpreted. As Oaks (1994: 378) states, lexical ambiguity is "a word with more than one possible meaning in a context."

The second type of ambiguity, syntactic ambiguity occurs in a sentence level. This ambiguity occurs because of the structure of the sentence. On the surface, syntactic jokes depend on "a duality of interpretation motivated by the structural patterns of the language system" (Lew, 1996 p.128) and ambiguity not of any lexical item but of the sentence of the syntactic level (Attardo et al. 1994b, p.35). The example of syntactic ambiguity is the ambiguous meanings of the sentence:

(ii) I shot an elephant in my pajama.

This sentence can have more than one interpretation. In the first interpretation, the sentence is interpreted as 'In my pajama, I shot an elephant' and in the second interpretation it is interpreted as ' $I$ shot an elephant (which is) wearing my pajama or (which is) in my pajama'. The multiple interpretations are caused by the structure of the sentence. This ambiguity is not caused by the meaning of the lexeme, but it is because of how the words are structured in the sentence. 'In my pajama' can either modify 'I' or 'an elephant' as a reduced clause. The structure does not violate any grammatical/ syntactic requirements and both interpretations have a solid ground. Since the ambiguity is caused by the structure/ syntax of the sentence, thus the ambiguity is classified into syntactic ambiguity.

\section{Lexical and Syntactic Ambiguity Overlap}

The lexical and syntactic ambiguity can overlap when there is word class change. Word class change occurs in lexical level, yet the word exhibit different syntactic functions, and thus has different meanings which invoke multi interpretations. To cope with the boundary fuzziness in this study, 'syntactic ambiguity resolution' proposed by MacDonald et al. (1994) is employed. In his proposed model, he states that "lexical and syntactic information in sentence comprehension is governed by common lexical processing mechanism and syntactic ambiguities are based on ambiguities in lexical level" (1994, p.682). Chiaro (1992) also places word class change in the lexical realm, while distinguishing syntactic ambiguity based on not any single of lexical item, but of (parts of) sentences at the syntactic level. Thus, in this study, the ambiguity caused by the different word class will be classified into lexical ambiguity. However, for the purpose of this study, compound nouns and noun phrase will be considered as syntactic ambiguity. An example of the lexical and syntactic ambiguity overlap is shown in the sentence below:

(iii) Reagan wins on budget, but no more lies ahead.

The ambiguity occurs in the use of 'lies' in the sentence above. Although the cause of the ambiguity involves only one lexicon, however, in this study the ambiguity is classified into syntactic ambiguity instead of lexical ambiguity. This is done by the consideration that the word 'lies' is ambiguous in this contexts because of the word category (noun-verb ambiguity) which leads to the different meaning. 


\section{Method}

The research is qualitative research. As stated by Fraenkel and Wallen (2009, p.422), in a qualitative study, the researcher is supposed to understand the holistic description of the phenomena. This research is a document-based study. Document or content analysis is a research method applied in written or visual materials (Ary et al, 2010, p.457). The data that were analyzed consist of 30 ambiguous sentences occurring in newspaper headlines, advertisement slogans, and jokes all of which were taken from electronic sources. The data were taken from various internet sites, as follows:

http://linguisticanthropology.org/blog/2013/12/09/ambiguity-jokes-and-trickquestions/, http://www.fun-with-words.com/ambiguous headlines.html, http://www.quora.com/What-are-some-great-lexical-ambiguity-jokes, http://www.cog.brown.edu/courses/cg7/11 ambiguity.pdf, http://www.alta.asn.au/events/altss w2003 proc/altss/courses/somers/headlines.ht

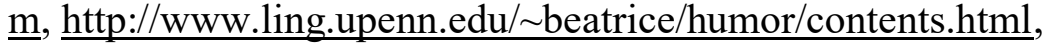
http://grammar.about.com/od/il/g/lexicalambiguityterm.htm, http://olgakagan.blog.com/2012/02/12/syntactic-ambiguity-in-cartoons/, and http://norvig.com/bls88.html.

The definition of the words is taken from Longman dictionary (Longman dictionary). The analysis is done through interpreting the possible multiple meanings in the sentences by describing the definition or the syntactic requirements in the contexts in which the ambiguity occurs.

\section{Findings and Discussion}

The collected data were analyzed; the data findings involved the classification of ambiguity and the analysis of meaning interpretations. From the total 25 data, 12 were lexically ambiguous while the 13 counterparts were syntactically ambiguous. The data were in form of sentences, or phrases taken form newspaper headlines, riddles and short story/ anecdotes. Each of the type of ambiguity is discussed respectively in this section including the analysis of how humorous meaning is created in each pun due to the dual interpretations

The findings of this study listed a total of 13 lexical ambiguities from the data collection in form of sentences and phrases. Some data were taken from newspaper headlines and advertisement slogans, therefore the data were not only in the form of sentences, but also phrases. However, both sentence and phrase forms in the data contain multiple meanings because of the ambiguity of the lexemes employed. The multiple interpretations result in the humorous elicitation.

(1) The hay farmer drank through a straw. (Taken from: http://norvig.com/bls88.html)

From the sentence above, the sentence is ambiguous because the word 'straw' has more than one meaning. Based on Longman dictionary, 'straw' can mean the dried stems of wheat or similar plants that animals sleep on, and that are used for making things, such as baskets, hat, etc. it is synonymous to hay. Another meaning of hay is a thin tube of paper or plastic for sucking up liquid from a bottle or a cup. These are two meanings of 'straw', which, especially in this context, create ambiguity. 
This sentence becomes ambiguously humorous because the subject of the sentence is 'the hay farmer'. Thus the sentence can mean either the farmer drank by using straw (plastic tube) or the farmer drank through straw (hay).

(2) Ross was told what to do by the river.

(Taken from: http://norvig.com/bls88.html)

The humor in this sentence is created by the ambiguous meaning of the word 'by'. The first meaning refers to the agent of the passive sentence and the second refers to the spatial information/ location. The noun 'the river' makes the ambiguous sentence humorous because it is not likely that the inanimate thing could tell Ross what to do. The serious meaning means that 'Ross was told what to do (by someone) by the river (showing the location)', while the humorous sentence means that 'Ross was told what to do by the river (the river is the agent of a passive sentence).'

(3) Prostitutes appeal to pope (newspaper headline)

(Taken from: http://www.fun-with-words.com/ambiguous headlines.html)

The ambiguous sentence is created by the word 'appeal'. 'Appeal' can be interpreted as 'to make a serious public request for help, money, information, etc' (Longman dictionary). However, appeal can mean 'be attractive (If someone or something appeals to you, they seem attractive and interesting)' (Longman dictionary). The serious meaning implies that prostitutes make a public requests to Pope, while the humorous meaning implies that Pope finds prostitutes attractive and interesting. What makes the sentence more hilarious is the subject and object of the sentence that is Pope and prostitutes.

(4) Stolen painting found by tree (newspaper headline)

(Taken from: http://www.fun-with-words.com/ambiguous headlines.html)

Similar to the case of sentence (2), the ambiguous meaning is caused because of the word 'by' which can be interpreted as the agent of passive sentence as well as the location information. The humorous meaning is created because it is unlikely that the tree found the stolen painting. This result also support the result of prior study on ambiguity and humor conducted by Bucaria (2004) which states that the humorous version of the meaning is created by the interpretation of 'by' as an agent of passive sentences instead of its intended spatial meaning.

(5) Miners refuse to work after death (newspaper headline)

(Taken from: http://www.fun-with-words.com/ambiguous headlines.html)

The humor is created because of the multiple interpretation of the word 'death'. 'Death' can be interpreted as 'the end of the life of a person' or 'a particular case when someone dies' (Longman dictionary). The serious meaning implies that the miners refuse to work after a particular case of someone's death. This is very possible that miners work with high risks that may cause accident. However, the humorous meaning implies that miners refuse to work after they die. 
This interpretation is humorous because it is impossible that they can work after they die.

(6) Take your mother-in-law out back and shoot her (Kodak advertisement). (Taken from: http://www.cog.brown.edu/courses/cg7/11_ambiguity.pdf)

The ambiguity is formed due two the dual meaning of the word 'shoot' that can be interpreted in this context. The first and intended meaning of the word 'shoot' is 'to take photographs or to make a film of something.' This definition is the intended meaning, since the sentence was taken from Kodak advertisement. The second possible and humorous meaning is 'to fire a gun/ to deliberately kill or injure someone using a gun' (Longman dictionary).

(7) Farmer Bill dies in House

(Taken from:

http://www.alta.asn.au/events/altss_w2003 proc/altss/courses/somers/head $\underline{\text { lines.htm) }}$

This sentence becomes ambiguous because of the words 'Bill' and 'House' which have more than one meaning. This context makes it possible for multi interpretations. 'Bill' can be interpreted as 'a name of a person' or 'a written proposal for a new law, that is brought to the parliament so that it can be discussed" (Longman dictionary). The second ambiguous word is 'House' which can mean 'a building where someone lives in' and 'a group of people who make the laws of a country, e.g. the House of Commons/ Representatives' (Longman dictionary). Thus, this sentence can mean that the Bill (written proposal) for farmer is declined by the House (parliament) when interpreted figuratively. On the other hand, it can also mean that farmer Bill dies in house (where he lives).

(8) Q: River Ravi flows in what state?

A: Liquid.

(Taken from: http://www.ling.upenn.edu/ beatrice/humor/contents.html)

This riddle is humorous because of the ambiguous meaning of the word 'state'. The answer of the question seems to be based on the different interpretation. The first, intended meaning of 'state' is country and the second meaning is 'condition/ physical or mental condition that someone or something is in' (Longman dictionary). Because of the different interpretation, the answer is not the expected answer of the question.

(9) "I have a really nice stepladder. Sadly, I never knew my real ladder." (English comedian Harry Hill)

(Taken from: http://grammar.about.com/od/il/g/lexicalambiguityterm.htm)

The ambiguous meaning of 'stepladder' is the source of humor in the sentence made by Harry Hill, an English comedian. 'Step-'has two possible meanings that can be used to interpret the sentence. The first meaning is 'stair/ a flat narrow piece of wood or stone, especially one in a series, that you put your 
foot on when you are up or down, especially outside a building (Longman dictionary). The second possible meaning is a prefix which is 'used to show that someone is related to you not by birth, but because a parent has married again (Longman dictionary). The second definition of step is the definition that is used to create a humor in this context. Therefore, the comedian stated 'I never know my real ladder.'

(10) She is looking for a match

(Taken from: http://grammar.about.com/od/il/g/lexicalambiguityterm.htm)

The ambiguity is created by the ambiguous meaning of the word 'match'. The word can be interpreted as 'a marriage' or 'a good opponent'. This sentence becomes humorous because of the different interpretations of the sentence.

(11) "You know, somebody actually complimented me on my driving today. They left a little note on the windscreen; it said, 'Parking Fine.' So that was nice."

(English comedian Tim Vine)

(Taken from: http://grammar.about.com/od/il/g/lexicalambiguityterm.htm)

The comedian creates the humor by the ambiguity that is formed from the word 'fine'. The first meaning refers to the noun which means 'money that you have to pay as a punishment' (Longman dictionary). The second meaning of the word 'fine' functions as an adjective, which means 'very good or a very high standard' (Longman dictionary). The humor is strengthened by the context in which the sentence occurs. The comedian, Tim Vine, led people to find humor in his mistaken interpretation in which he interpreted the parking fine (a form of punishment) as the compliment that somebody gave because he parked well. In terms of meaning, the punishment is the opposite of the complement, because punishment is given when someone does something wrong, meanwhile a compliment is given when someone does something very well. The meaning relation makes the humorous sense even stronger. Therefore, the ambiguity is used by the comedian to elicit humor.

(12) Iraqi head seeks arms (newspaper headline)

(Taken from: http://www.fun-with-words.com/ambiguous_headlines.html)

In this sentence, ambiguity is formed because of the multiple meanings of the word 'head' and 'arms'. 'Head' can have more than one meaning because of polysemous meaning relation. 'Head' literally can mean as 'the top part of your body that has your face at the front and is supported by your neck' (Longman dictionary). The second meaning refers to 'the front or the most important position/ leading position' (Longman dictionary). The second ambiguous word in the sentence is 'arms'. In its first meaning, 'arms' refer to 'the two long parts of your body between your shoulders and your hands' (Longman dictionary). The second meaning of 'arms' refer to 'weapons used for fighting wars' (Longman dictionary). 
The sentence can thus, be interpreted as 'an Iraqi leader seeks weapons' (the serious interpretation). However, because of the lexical ambiguity, this sentence can also be interpreted literally as 'an Iraqi head (the top part of human body) seeks arms (the part of human body).' The interpretation of the ambiguous meanings become humorous because of the meaning relations of 'head' and 'arms' in which both are the members of the same hypernym of a human body.

The findings of this study listed 18 data of syntactic ambiguities from the collection of sentences and phrases. These ambiguous sentences and phrases were taken from newspaper headlines, advertisement slogans and other electronic sources. The sentences elicit humorous senses due to the dual interpretations of each sentence. The discussion and analysis of each ambiguous sentence are as follows:

(13) A: I saw a man-eating shark at the aquarium.

B: That's nothing. I saw a man eating herring at the deli

(Taken from: http://linguisticanthropology.org/blog/2013/12/09/ambiguityjokes-and-trick-questions/)

The humor is created because of the ambiguity of the phrase 'a man-eating sharks' and 'a man eating herring'. These phrases can have dual interpretation due to its syntactic form. 'A man eating shark' can mean a man who eats sharks or sharks which eat a man. Both interpretations can be accepted in terms of meaning because those possibilities are likely to represent what the speaker intends to convey.

However, the same structure with only a different noun can create a humorous ambiguity. The humorous interpretation is strengthened by the B response 'a man eating herring' which can also be interpreted as 'a man who eats herring' or 'herring (small fish) which eats a man.' This ambiguity becomes the source of humor because from the same sentence with this structure, the interpretations can be very different, yet both interpretations are based on the way the sentence is structured.

(14) Complaints about NBA referees growing ugly (newspaper headline)

(Taken from: http://www.fun-with-words.com/ambiguous headlines.html)

This headline is humorous because of the ambiguity in the phrase. The structure of the phrase makes it possible to be interpreted in two different meanings. The interpretation, which is the serious intended interpretation, describes the complaint (about the NBA referees) which grows ugly, meaning that the complaint becomes worse or bigger. However, it may also be humorously interpreted that the complaint is about NBA referees who grow ugly.

(15) Dealers will hear car talk at noon (newspaper headline)

(Taken from: http://www.fun-with-words.com/ambiguous_headlines.html)

The sentence above can be ambiguous because of the way the sentence is structured, especially in the phrase 'car talk'. The arrangement of the sentence makes the sentence have two possible meanings and interpretations. The first 
interpretation of car talk is the talk about cars, as in the noun phrase. The second interpretation, however, implies that dealers will hear car talk in which the car is an agent which can talk. This interpretation is humorous given that the car, as an inanimate object is not likely to talk. The result of the analysis is also in line with the finding of the previous study which analyzed the same data by Bucaria (2004). In the paper Bucaria states that the humorous meaning occurs because of the possibility of interpreting 'talk' as a noun or a verb.

(16) Drunken drivers paid $\$ 1,000$ in '84 (newspaper headline)

(Taken from: http://www.fun-with-words.com/ambiguous headlines.html)

In this sentence, the ambiguity lies in the possible meaning of the word 'paid'. 'Paid' can either be the past tense of 'pay' or the past participle of 'pay'. The difference can lead to the construction of an active or a passive sentence. Although the ambiguity is mainly caused by the meaning of a word, that is 'pay', in this sentence. The ambiguity is classified into syntactic ambiguity, based on the consideration that is discussed in the previous discussion. The ambiguity is created because the word belongs to different categories or syntactic function in the sentence. The different syntactic possible functions of the word make it possible for the sentence to be interpreted in an active or passive construction.

(17) Man eating piranha mistakenly sold as pet fish (newspaper headline)

(Taken from: http://www.fun-with-words.com/ambiguous headlines.html)

The ambiguous sentence becomes humorous because the phrase 'mistakenly sold as pet fish' structurally possible to explain both the 'man' and 'piranha.' The serious, intended meaning describes a man who eats piranha which is mistakenly sold as pet fish, whereas the humorous interpretation implied that the man who eats piranha is mistakenly sold as a pet fish.

(18) Include your children when baking cookies (newspaper headline)

(Taken from: http://www.fun-with-words.com/ambiguous headlines.html)

In this sentence the humorous meaning is created because the sentence in form of imperative sentence above can be interpreted as a command to 'include your children when you bake cookies' and it can also be interpreted as 'you should include your children in the cookies that you bake.' This humorous interpretation can be made because of the verb 'include' and the reduced form after 'when', which can mean that 'you should include your children (in the time) when you bake cookies' or 'include your children in the cookies when (if) you bake.'

(19) Squad helps dog bite victim (newspaper headline)

(Taken from: http://www.fun-with-words.com/ambiguous headlines.html)

This sentence is ambiguous because of the way words in the phrase 'helps dog bite victim.' Syntactically, following the use of the word 'help', an infinitive verb can be used. 'Help' can be followed by either a direct object or an infinitival 
complement (a reduced form, in this case). Thus, the sentence can be interpreted in two ways: the first, which is the serious intended meaning, is 'Squad helps the victim of dog bite'. The phrase is in the form of noun phrase. The second, humorous interpretation means 'squad helps dog to bite victim.' This ambiguity is also formed by the possible function of 'bite' as a noun and verb.

(20) Stud tires out (newspaper headline)

(Taken from: http://www.fun-with-words.com/ambiguous headlines.html)

The sentence becomes ambiguous, especially because there is a different spelling of English and American English. Allowing the American spelling of 'tyres' (in British English it is usually known as 'studded tyres'). The ambiguity is structural because the of the noun-verb ambiguity of 'tyres'. This ambiguity becomes more confusing due to the lexical ambiguity of 'stud'. Based on Longman dictionary, 'stud' can mean 'the use of animal, especially horses for breeding. It can also mean 'a round piece of metal that is stuck into a surface for decoration. It is particularly confusing because typically, a newspaper headline does not necessarily include the main verb.

(21) Hospitals are sued by 7 foot doctors (newspaper headline)

(Taken from: http://www.fun-with-words.com/ambiguous headlines.html)

The ambiguity lies in the ' 7 foot doctors' noun phrase. This phrase can be interpreted as 7 'doctors who are specialized in foot' or doctors who are 7 feet tall. The syntactic requirement of a noun phrase requires a singular form of foot as in 'a 3 year-old child.' Thus, the sentence is syntactically ambiguous.

(22) The village blacksmith finally found an apprentice willing to work hard for long hours. The blacksmith immediately began his instructions to the lad, "When I take the shoe out of the fire, I'll lay it on the anvil; and when I nod my head, you hit it with this hammer."

The apprentice did just as he told. Now he's the village blacksmith.

(Taken from: http://www.quora.com/What-are-some-great-lexicalambiguity-jokes)

The joke is created because of the dual interpretation of pronoun 'it' in '... you hit it with this hammer.' Two nouns which can be replaced by the pronoun 'it' are previously stated; they include 'the shoe' and 'my head.' In this anecdote, the listener misinterprets what the blacksmith says and hits the blacksmith's head instead of the shoe. This ambiguity occurs due to referential ambiguity represented by the pronoun.

(23) Come meet our new French pastry chef.

(Taken from: http://www.cog.brown.edu/courses/cg7/11 ambiguity.pdf)

The sentence is ambiguous because of the noun phrase 'French pastry chef', which can mean 'a chef who is French' or 'a chef of French pastry.' 
(24) One morning I shot a huge lion in my pajama

(Taken from: http://olgakagan.blog.com/2012/02/12/syntactic-ambiguityin-cartoons/)

The sentence is ambiguous because or the prepositional phrase 'in my pajama', which can function to modify the noun 'I' or 'a huge lion.' The ambiguity can create humor especially because in its humorous interpretation, a huge lion can be in one's pajama.

(25) The chicken is ready to eat

(Taken from: http://olgakagan.blog.com/2012/02/12/syntactic-ambiguityin-cartoons/)

This structure often causes ambiguity. In English one can say, for example, 'the book is hard to understand' to convey the meaning the content of the book is complex or confusing. It is not necessary to say 'the book is hard to be understood'. In this context, therefore, the sentence can mean either 'the chicken is served' or 'the chickens are ready to be fed or to eat something.'

\section{Conclusion}

From the analysis of the ambiguous contexts, humors are created because the ambiguity offers possible dual interpretations that the reader can conclude from the sentences. The types of ambiguity that can form puns in humors are lexical and syntactic ambiguity. Lexically, the multi lexical meanings can confuse the reader and at the same time can create a mind-blowing possible interpretation, and thus can be a language device used to create puns in humor. Syntactically, the syntactic requirements and the way the words are structured in the sentence can also create ambiguity. In details, syntactic ambiguity can be forms by the syntactic requirement based on its functions and forms, such as ambiguity in prepositional phrase of the sentence, noun phrase, active-passive construction, pronouns, different word categories which lead to different meanings, etc.

The ambiguity can strengthen the humorous meaning especially when the possible interpretations involve a serious meaning and a humorous counterpart. The contexts and the choice of words also influence the process of how the humor is created. Ambiguity can occur only in certain contexts with relevant choice or words or with certain syntactic orders (by also considering the syntactic requirements). Thus, the contexts play an important function to make it possible for the sentences to be interpreted in different ways.

\section{References}

Anatonopoulou, E. (2004). Humor theory and translation research: Proper names in humorous discourse. Humor, 17(3), 219-255.

Ary, D., Jacobs, L. C., \& Soronsen, C. (2010). Introduction to research in education ( $8^{\text {th }}$ ed.). Belmont, CA: Wadsworth Cengage Learning.

Attardo, S. (1994a). Linguistic theories of humor. Berlin: Walker de Gruyter.

Attardo, S., Attardo, D.H., Baltes, P., \& Petray, M.J. (1994b). The linear organization of jokes: Analysis of two thousand texts. Humor, 7(1), 27-54. 
Bucaria, C. (2004). Lexical and syntactic ambiguity as a source of humor: The case of newspaper headlines. Humor, 17(3), 279-309.

Chiaro, D. (1992). The language of jokes: Analysing verbal play. London: Routledge.

Duffy, S., Kambe, G., \& Rayner, K. (2001). The affect of prior disambiguating context on the comprehension of ambiguous words: evidence from eye movements. In D.S. Gorfein (Ed.), On the consequences of meaning selection: Perspectives on resolving lexical ambiguity (pp. 27-43). Washington, D.C.: American Psychological Association.

Fraenkel, J. R., \& Wallen, N. E. (2009). How to design and evaluate research in education $\left(7^{\text {th }}\right.$ ed.). New York: McGraw-Hill.

Gibson, E. \& Pearlmutter, N. (1994) A corpus-based analysis of psycholinguistic constraints on prepositional phrase attachment. In Clifton, Charles Jr., Lyn Frazier, and Keith Rayner (eds.), Perspectives on sentence processing. Hillsdale, NJ: Lawrence Erlbaum Associates, 181-198.

Giora, R. (2003). On our mind. salience, context, and figurative language. Oxford: Oxford University Press.

Gorfein, D.S. (2001). On the consequences of meaning selection: an overview. In D.S. Gorfein (Ed.), On the consequences of meaning selection: Perspectives on resolving lexical ambiguity. Washington, D.C.: American Psychological Association.

Hirst, G. (1987). Semantic interpretation and the resolution of ambiguity. Cambridge: Cambridge University Press.

Kreidler, C. (2002). Introducing English semantics. London: Routledge.

Laurian, A. (1992). Possible/impossible translation of jokes. Humor 3(1/2), 111127.

Lew, R. (1996). Exploitation of linguistic ambiguity in Polish and English jokes. Papers and Studies in Contrastive Linguistics, 31, 127-133.

Longman Dictionary of Contemporary English. (2009) $\left(5^{\text {th }}\right.$ ed.). Essex: Pearson Education Limited.

MacDonald, M.C., Pearlmutter, N.J., \& Seidenberg, M.S. (1994). Lexical nature of syntactic ambiguity resolution. Psychological Review, 4, 676-703.

Oaks, D.D. (1994). Creating structural ambiguities in humor: Getting English grammar to cooperate. Humor, 7(4), 377-401.

Ptaszynski, M. \& Mickiewicz, A. (2004). On the (un)translatability of jokes. Perspectives: Studies in Translatology, 12(3), 176-193.

Seewoester, S. (2009). Linguistic ambiguity in language-based jokes. College of Communication M.A. Thesis. Retrieved on March 15, 2016, from http://via.library.depaul.edu/cmnt/3

Tabossi, P. (1988). Accessing lexical ambiguity in different types of sentential contexts. Journal of Memory and Language, 27(3), 324-340.

Zabalbeascoa, P. (1996). Translating jokes for dubbed television situation comedies. The Translator, 2(2), 235-257. 